Europhys. Lett., 64 (1), pp. 29-35 (2003)

\title{
Dynamics and steady state of a vibrated granular binary mixture model
}

\author{
A. Prados and J. J. Brey \\ Físice Teórica, Universidad de Sevilla, Apartado de Correos 1065 \\ E-41080 Sevilla, Spain
}

(received 2 May 2003; accepted in final form 2 August 2003)

PACS. 45.70.-n - Granular systems.

PACS. 05.50.+q - Lattice theory and statistics (Ising, Potts, etc.).

PACS. 45.70.Mg - Granular flow: mixing, segregation and stratification.

\begin{abstract}
A model for the dynamical evolution of a granular binary mixture is analyzed. This system is submitted to a tapping procedure, similarly to what is done in real experiments. In the weak vibration limit, an effective dynamics for the tapping process is derived, and the steady-state probability distribution is analytically found. The steady probability does not depend on the details of the configuration, but only on the number of particles of each of the two species. Depending on the values of their fugacities, the system can be either almost full of small or big particles, i.e., segregation effects are present.
\end{abstract}

Granular systems have attracted the attention of physicists in recent years. A review of some of the basic phenomenological features of granular matter can be found in refs. [1,2]. Many of these behaviours, like compaction [3] or segregation [4,5], are far from being well understood. In statistical physics, simple models are often used as a first approximation to many different, complex, problems. Thus, recently, several Ising or particle-hole models [6-11] have been used to analyze compaction processes in dense granular media. Also, the parking lot model has been employed in the same context [12].

One of the most interesting physical questions in the physics of dense granular media is the description of the steady state eventually reached by a system, when it is externally perturbed. Thermodynamics is not directly applicable to powders. Nevertheless, some years ago, Edwards and coworkers [13] made the hypothesis that the steady state of an externally perturbed granular system can be described by an extension of the usual statistical-mechanics concepts. The central point is the ergodic hypothesis for externally perturbed powders: in the steady state, all the mechanically stable (metastable) configurations of a granular assembly occupying the same volume have the same probability.

When a granular mixture is shaken, their components often tend to separate, leading to the well-known phenomenon of segregation [1,2]. Usually, when a bidisperse granular system is vibrated, larger particles go to the top, the so-called Brazil Nut effect (BNE) [4,5,14]. Very recently, it has been found that, as the mass density of the big grains is increased as compared with that of the small grains, this phenomenon can be inverted: small grains go to the top 
while large grains move to the bottom of the container $[15,16]$. This is known as the reverse Brazil Nut effect (RBNE).

One of the main purposes of this paper is trying to understand some aspects of the dynamical behaviour of binary granular mixtures by means of the analysis of a simple, analytically tractable, model. In particular, we will be interested in the study of the steady state reached by the system in the long-time limit. Its relationship with Edwards' theory will be discussed, and also the appearance of both the BNE and RBNE behaviours.

Let us consider a horizontal section of a real granular binary mixture, near the bottom of its container. During the free evolution of the system, i.e., only under the action of gravity, particles can only go down, as long as there is enough empty space in their surroundings. The total density of particles in the layer grows until the hard-core interaction prevents more movements of particles, and a metastable (mechanically stable) configuration is reached. On the other hand, when the system is submitted to vertical vibration, particles can go up, decreasing the density in the layer. In both processes, big particles will need more free space in their surroundings than small particles to be adsorbed on or desorbed from the layer.

We introduce a one-dimensional lattice of $N$ sites, which can be either empty (occupied by a hole), occupied by a small particle A, or occupied by a big particle B. Variables $n_{i}$ and $m_{i}$ are defined as follows. If site $i$ is occupied by a particle $\mathrm{A}, n_{i}=0$, otherwise $n_{i}=1$. If site $i$ is occupied by a particle $\mathrm{B}$, then $m_{i}=0$, if not, $m_{i}=1$. Then, an empty site is given by $n_{i}=m_{i}=1$. The numbers $1-n_{i}$ and $1-m_{i}$ are the occupation numbers of particles A and B, respectively. The specification of $\boldsymbol{n}=\left\{n_{i}\right\}$ and $\boldsymbol{m}=\left\{m_{i}\right\}$ characterizes a configuration of the lattice. The dynamics of the system is assumed to be a Markov process defined by the following master equation for the probability $p(\boldsymbol{n}, \boldsymbol{m}, t)$ for finding the system in the configuration $\{\boldsymbol{n}, \boldsymbol{m}\}$ at time $t$ :

$$
\begin{aligned}
\partial_{t} p(\boldsymbol{n}, \boldsymbol{m}, t)= & \sum_{i}\left[W_{i}\left(\boldsymbol{n}, \boldsymbol{m} \mid R_{i} \boldsymbol{n}, \boldsymbol{m}\right) p\left(R_{i} \boldsymbol{n}, \boldsymbol{m}, t\right)-W_{i}\left(R_{i} \boldsymbol{n}, \boldsymbol{m} \mid \boldsymbol{n}, \boldsymbol{m}\right) p(\boldsymbol{n}, \boldsymbol{m}, t)\right]+ \\
& +\sum_{i}\left[W_{i}\left(\boldsymbol{n}, \boldsymbol{m} \mid \boldsymbol{n}, R_{i} \boldsymbol{m}\right) p\left(\boldsymbol{n}, R_{i} \boldsymbol{m}, t\right)-W_{i}\left(\boldsymbol{n}, R_{i} \boldsymbol{m} \mid \boldsymbol{n}, \boldsymbol{m}\right) p(\boldsymbol{n}, \boldsymbol{m}, t)\right] .
\end{aligned}
$$

Here, $R_{i} \boldsymbol{m}=\left\{\ldots, m_{i-1}, 1-m_{i}, m_{i+1}, \ldots\right\}$ and $R_{i} \boldsymbol{n}=\left\{\ldots, n_{i-1}, 1-n_{i}, n_{i+1}, \ldots\right\}$. Thus, the possible events are the adsorption of an A or B particle on an empty site, and their desorption from an occupied site. In order to model the hard-core interactions, a facilitated dynamics [17] is considered. Small particles A need, to be adsorbed on or desorbed from a site of the lattice, at least one of its two nearest-neighbour sites being empty. Namely,

$$
W_{i}\left(R_{i} \boldsymbol{n}, \boldsymbol{m} \mid \boldsymbol{n}, \boldsymbol{m}\right)=\left[\frac{\alpha_{\mathrm{a}}}{2} n_{i} m_{i}+\frac{\alpha_{\mathrm{d}}}{2}\left(1-n_{i}\right) m_{i}\right]\left(n_{i-1} m_{i-1}+n_{i+1} m_{i+1}\right),
$$

where $\alpha_{\mathrm{a}}$ and $\alpha_{\mathrm{d}}$ are the characteristic rates for the attempts of adsorption and desorption of particles A, respectively. On the other hand, big particles B need both of its nearest neighbours empty when adsorbing on a site or desorbing from it,

$$
W_{i}\left(\boldsymbol{n}, R_{i} \boldsymbol{m} \mid \boldsymbol{n}, \boldsymbol{m}\right)=\left[\beta_{\mathrm{a}} n_{i} m_{i}+\beta_{\mathrm{d}} n_{i}\left(1-m_{i}\right)\right] n_{i-1} m_{i-1} n_{i+1} m_{i+1},
$$

$\beta_{\mathrm{a}}$ and $\beta_{\mathrm{d}}$ being the rates for the attempts of particles $\mathrm{B}$ to adsorb on and desorb from the lattice, respectively. If particles $\mathrm{B}$ are eliminated, i.e., $m_{i}=1$ for all $i$ and $\beta_{\mathrm{a}}=0$, our system reduces to a model for a single type of particles $[6,18]$.

We are interested in the particularization of the above general dynamics for the tapping process used in the laboratory to vibrate real granular systems [3]. It will be modelled as 


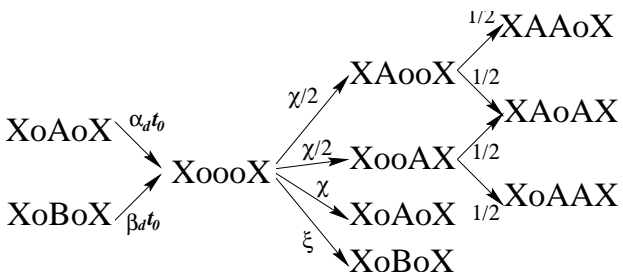

Fig. 1

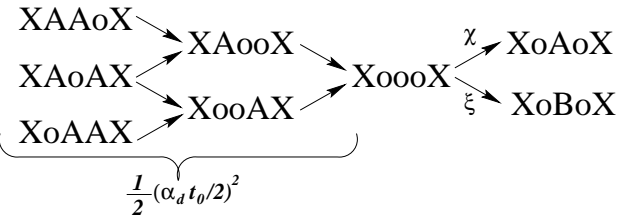

Fig. 2

Fig. 1 - Desorption of a particle between two empty sites, followed by the events during the free relaxation necessary to get into a metastable configuration.

Fig. 2 - Second-order processes being the inverse of those first-order trajectories increasing the density of the system. Two desorption events during the pulse of duration $t_{0}$ are needed.

follows, analogously to the procedure introduced for a monodisperse system [6,8]. Starting from an empty lattice, the system is allowed to relax with $\alpha_{\mathrm{d}}=\beta_{\mathrm{d}}=0$, i.e., only adsorption events are allowed. The system evolves until it reaches a metastable configuration, in which no more adsorptions are possible. The metastable configuration so obtained will be the initial state for the tapping process and will be referred to as state $r=0$. Next, we describe the vibration process by making $\alpha_{\mathrm{a}}=\beta_{\mathrm{a}}=0$, particles can only desorb from the lattice, for a given time interval $t_{0}$. This pulse takes, in general, the system out from the metastable configuration. Afterwards, the system relaxes again with $\alpha_{\mathrm{d}}=\beta_{\mathrm{d}}=0$ until it gets stuck in a new metastable configuration $r=1$. By repeating this sequence, a series of metastable states $r=2,3,4, \ldots$ is generated. The index $r$ indicates the number of taps made before that metastable configuration has been reached. Notice that the non-conservation of the number of particles in the lattice tries to mimic what actually occurs in a deep layer of a vibrated granular system.

The metastable configurations are characterized as follows: i) all the holes are isolated, ii) two big particles are separated by a domain of sites of length $l \geq 1$ and, moreover, that domain cannot be completely full of particles A. Thus, the number of holes verifies $N_{\mathrm{H}}=$ $N-N_{\mathrm{A}}-N_{\mathrm{B}}>N_{\mathrm{B}}$. The number of metastable configurations $\Omega_{N_{\mathrm{A}}, N_{\mathrm{B}}}^{(N)}$ for a lattice of $N$ sites with $N_{\mathrm{A}}$ particles $\mathrm{A}$ and $N_{\mathrm{B}}$ particles B is [19]

$$
\Omega_{N_{\mathrm{A}}, N_{\mathrm{B}}}^{(N)}=\frac{\left(N-N_{\mathrm{A}}-N_{\mathrm{B}}-1\right) !\left(N_{\mathrm{A}}+2 N_{\mathrm{B}}+1\right) !}{N_{\mathrm{B}} !\left(N-N_{\mathrm{A}}-2 N_{\mathrm{B}}-1\right) !\left(2 N_{\mathrm{A}}+2 N_{\mathrm{B}}-N+1\right) !\left(N-N_{\mathrm{A}}\right) !} .
$$

In the large- $N$ limit, there will be well-defined densities of particles $\mathrm{A}$ and $\mathrm{B}, \rho_{\mathrm{A}} \equiv N_{\mathrm{A}} / N$ and $\rho_{\mathrm{B}} \equiv N_{\mathrm{B}} / N$, and the number of states $\Omega_{N_{\mathrm{A}} N_{\mathrm{B}}}^{(N)}$ is exponentially large. Thus, $\ln \Omega_{N_{\mathrm{A}} N_{\mathrm{B}}}^{(N)} / N$ is only a function of $\rho_{\mathrm{A}}$ and $\rho_{\mathrm{B}}$ and, therefore, independent of $N$ itself, i.e., the "microcanonical" entropy $S \equiv \ln \Omega_{N_{\mathrm{A}} N_{\mathrm{B}}}^{(N)}$ is an extensive property.

The chain of metastable configurations obtained by the tapping procedure defines a Markov process: no information is needed from the metastable state $r-1$ in order to compute the probability of going from configuration $r$ to $r+1$. This is due to the free relaxation occurring between every two vibration pulses. Therefore, it is tempting to try to identify the transition probabilities $W_{\text {ef }}\left(\boldsymbol{n}^{\prime}, \boldsymbol{m}^{\prime} \mid \boldsymbol{n}, \boldsymbol{m}\right)$ from the initial metastable configuration $\{\boldsymbol{n}, \boldsymbol{m}\}$ to the final one $\left\{\boldsymbol{n}^{\prime}, \boldsymbol{m}^{\prime}\right\}$ in a single tap. This can be done if $\alpha_{\mathrm{d}} t_{0} \ll 1, \beta_{\mathrm{d}} t_{0} \ll 1$, i.e., the probability for the desorption events during the vibration is very small. Then, an expansion in powers of $\alpha_{\mathrm{d}} t_{0}$ and $\beta_{\mathrm{d}} t_{0}$ is expected to be useful.

The lowest order is equivalent to consider that each site is affected by only one rearrangement in a single tap. Due to the facilitated dynamics, only particles A next to at least one 
TABLE I - Transitions between metastable states, in a single tap, with their corresponding rates.

\begin{tabular}{cccc}
\hline First order & Initial state & Final state & $W_{\mathrm{ef}}$ \\
\hline one-site diffusion & XAoX & XoAX & $\alpha_{\mathrm{d}} t_{0} / 4$ \\
& XoAX & XAoX & $\alpha_{\mathrm{d}} t_{0} / 4$ \\
change B into A & XoBoX & XoAoX & $\beta_{\mathrm{d}} t_{0} \chi$ \\
change A into B & XoAoX & XoBoX & $\alpha_{\mathrm{d}} t_{0} \xi$ \\
change B into 2A & XoBoX & XAoAX & $\beta_{\mathrm{d}} t_{0} \chi / 2$ \\
& XoBoX & XAAoX or XoAAX & $\beta_{\mathrm{d}} t_{0} \chi / 4$ \\
change A into 2A & XoAoX & XAoAX & $\alpha_{\mathrm{d}} t_{0} \chi / 2$ \\
& XoAoX & XAAoX or XoAAX & $\alpha_{\mathrm{d}} t_{0} \chi / 4$ \\
\hline Second order & Initial state & Final state & $W_{\mathrm{ef}}$ \\
\hline change 2A into B & XAAoX or XoAAX & XoBoX & $\left(\alpha_{\mathrm{d}} t_{0}\right)^{2} \xi / 8$ \\
& XAoAX & XoBoX & $\left(\alpha_{\mathrm{d}} t_{0}\right)^{2} \xi / 4$ \\
change 2A into A & XAAoX or XoAAX & XoAoX & $\left(\alpha_{\mathrm{d}} t_{0}\right)^{2} \chi / 8$ \\
& XAoAX & XoAoX & $\left(\alpha_{\mathrm{d}} t_{0}\right)^{2} \chi / 4$ \\
\hline
\end{tabular}

empty site or particles B between two empty sites can desorb, leading the system to an unstable configuration. Afterwards, the free relaxation will take the system back to a metastable configuration. Let us consider a small particle A next to only one hole. It desorbs during the vibration with probability $\alpha_{\mathrm{d}} t_{0} / 2$. Afterwards, in the free relaxation, a small particle $\mathrm{A}$ is adsorbed in any of the two neighbouring empty sites with the same probability $1 / 2$. Therefore, the probability for a one-site diffusion process of small particles $\mathrm{A}$ is $\alpha_{\mathrm{d}} t_{0} / 4$, as shown in table I. There, the symbol o represents an empty site, and X stands for an occupied site, either by a particle $\mathrm{A}$ or a particle $\mathrm{B}$. On the other hand, there are no diffusive processes for particles B up to this order, since they need both of their nearest neighbours empty in order to desorb. The other lowest-order processes start with the desorption of a particle between two empty sites. The possible trajectories are shown in fig. 1, in which the probability for each step is given. We have introduced the parameters $\chi=\alpha_{\mathrm{a}} /\left(2 \alpha_{\mathrm{a}}+\beta_{\mathrm{a}}\right)$ and $\xi=\beta_{\mathrm{a}} /\left(2 \alpha_{\mathrm{a}}+\beta_{\mathrm{a}}\right)$. The transition probabilities for each rearrangement connecting metastable states is obtained as the product of the probabilities for each of the steps composing it, and they are summarized in table I. We do not compute the probabilities for the transitions leading to a final state identical to the initial one, since they are not needed when using a master equation description [20]. To the lowest order, only compaction events take place, since the total number of particles $N_{\mathrm{A}}+N_{\mathrm{B}}$ always increases. In order to have processes decreasing the total density, we are led to consider second-order terms, similarly to what happened in the monodisperse case $[6,10]$. In particular, we will analyze whether the inverse processes of those increasing the density to the lowest order are possible, and which are their transition probabilities. These are depicted in fig. 2, together with the probability of each elementary step. The corresponding effective transition probabilities between metastable configurations are also included in table I. The Markov process defined by the effective transition rates of table I is irreducible, i.e., all the metastable configurations are connected by a chain of transitions with non-zero probability. This assures the existence of a unique stationary solution of the master equation.

In order to derive the steady-state distribution, we will bet a priori on a stationary solution $P^{(\mathrm{s})}(\boldsymbol{n}, \boldsymbol{m})$ of the master equation having the detailed balance property,

$$
W_{\mathrm{ef}}\left(\boldsymbol{n}, \boldsymbol{m} \mid \boldsymbol{n}^{\prime}, \boldsymbol{m}^{\prime}\right) P^{(\mathrm{s})}\left(\boldsymbol{n}^{\prime}, \boldsymbol{m}^{\prime}\right)=W_{\mathrm{ef}}\left(\boldsymbol{n}^{\prime}, \boldsymbol{m}^{\prime} \mid \boldsymbol{n}, \boldsymbol{m}\right) P^{(\mathrm{s})}(\boldsymbol{n}, \boldsymbol{m}) .
$$

Let us consider, first, metastable states without particles B, i.e., $N_{\mathrm{B}}=0$. All the states with 
the same number of particles A must have the same probability in the steady state, since they are connected through (isotropic) diffusive processes. This means that any metastable state has a steady probability which only depends on the values of $N_{\mathrm{A}}$ and $N_{\mathrm{B}}$, because any metastable configuration can be obtained from a configuration with $N_{\mathrm{B}}=0$ through a chain of diffusive processes and $N_{\mathrm{B}}$ substitutions of particles A by particles B. Then, application of detailed balance to the processes in table I yields, for the steady probability of any metastable state with $N_{\mathrm{A}}$ particles $\mathrm{A}$ and $N_{\mathrm{B}}$ particles B,

$$
P_{N_{\mathrm{A}} N_{\mathrm{B}}}^{(\mathrm{s})}=\frac{1}{Z} \gamma_{\mathrm{A}}^{-N_{\mathrm{A}}} \gamma_{\mathrm{B}}^{-N_{\mathrm{B}}}, \quad \gamma_{\mathrm{A}}=\frac{1}{2} \alpha_{\mathrm{d}} t_{0}, \quad \gamma_{\mathrm{B}}=\frac{\alpha_{\mathrm{a}}}{2 \beta_{\mathrm{a}}} \beta_{\mathrm{d}} t_{0},
$$

and $Z$ is a generalized "partition function",

$$
Z=\sum_{N_{\mathrm{A}}} \sum_{N_{\mathrm{B}}} \Omega_{N_{\mathrm{A}} N_{\mathrm{B}}}^{(N)} \gamma_{\mathrm{A}}^{-N_{\mathrm{A}}} \gamma_{\mathrm{B}}^{-N_{\mathrm{B}}}
$$

All the states with the same numbers $N_{\mathrm{A}}$ and $N_{\mathrm{B}}$ have the same probability; this is consistent with Edwards' thermodynamic description of the steady state of externally perturbed powders [13]. As we are dealing with an open system having two different kinds of particles, the steady state is described by two thermodynamic parameters, the "fugacities" $\gamma_{\mathrm{A}}^{-1}$ and $\gamma_{\mathrm{B}}^{-1}$ corresponding to particles $\mathrm{A}$ and $\mathrm{B}$, respectively.

In the large- $N$ limit, the partition function is readily obtained by means of the saddle point method, with the result [19]

$$
\ln \zeta \equiv \frac{1}{N} \ln Z=\ln \frac{2 \rho_{\mathrm{B}}+\rho_{\mathrm{A}}}{\left(2 \rho_{\mathrm{A}}+2 \rho_{\mathrm{B}}-1\right) \gamma_{\mathrm{A}}},
$$

where $\rho_{\mathrm{A}}$ and $\rho_{\mathrm{B}}$ are the steady densities of particles $\mathrm{A}$ and $\mathrm{B}$, respectively, being $0 \leq \rho_{\mathrm{A}} \leq 1$ while $0 \leq \rho_{\mathrm{B}} \leq 1 / 2$, since there must be at least one hole between every two particles $\mathrm{B}$. Moreover, $\rho_{\mathrm{H}}=1-\rho_{\mathrm{A}}-\rho_{\mathrm{B}} \geq \rho_{\mathrm{B}}$. The particle densities are determined by the saddle point condition

$$
\gamma_{\mathrm{A}}=\frac{\left(\rho_{\mathrm{A}}+2 \rho_{\mathrm{B}}\right)\left(1-\rho_{\mathrm{A}}-2 \rho_{\mathrm{B}}\right)\left(1-\rho_{\mathrm{A}}\right)}{\left(1-\rho_{\mathrm{A}}-\rho_{\mathrm{B}}\right)\left(2 \rho_{\mathrm{A}}+2 \rho_{\mathrm{B}}-1\right)^{2}}, \quad \gamma_{\mathrm{B}}=\frac{\left(\rho_{\mathrm{A}}+2 \rho_{\mathrm{B}}\right)^{2}\left(1-\rho_{\mathrm{A}}-2 \rho_{\mathrm{B}}\right)^{2}}{\rho_{\mathrm{B}}\left(1-\rho_{\mathrm{A}}-\rho_{\mathrm{B}}\right)\left(2 \rho_{\mathrm{A}}+2 \rho_{\mathrm{B}}-1\right)^{2}},
$$

which give $\rho_{\mathrm{A}}$ and $\rho_{\mathrm{B}}$ in terms of the fugacities. The situation is, in this sense, analogous to the one found in a recent analysis of a hard-sphere mixture, where an extension of Edwards' theory with two different values of the compactivity for each of the species was introduced [21]. As explained above, in our model two thermodynamic parameters are present because we are dealing with an open system. Thus, as in molecular systems, two "fugacities" are needed, one for each kind of particles.

In the weak vibration limit $\gamma_{\mathrm{A}} \ll 1, \gamma_{\mathrm{B}} \ll 1$ we are considering, an asymptotic analysis, up to order $\gamma_{\mathrm{A}}$, gives

$$
\rho_{\mathrm{A}}=\frac{1}{1+\eta}-\frac{1}{2} \frac{1-\eta}{(1+\eta)^{3}} \gamma_{\mathrm{A}}, \quad \rho_{\mathrm{B}}=\frac{1}{2} \frac{\eta}{1+\eta}-\frac{1}{2} \frac{\eta}{(1+\eta)^{3}} \gamma_{\mathrm{A}},
$$

uniformly valid for $\gamma_{\mathrm{B}} \ll 1$ [19]. In eq. (10), it is $\eta=\gamma_{\mathrm{A}} / \sqrt{\gamma_{\mathrm{B}}}$. Figure 3 compares the asymptotic values of the densities, as given by eq. (10), and the numerics, obtained by Monte Carlo simulation, for $\gamma_{\mathrm{A}}=\gamma_{\mathrm{B}}$. The agreement is quite good up to $\gamma_{\mathrm{A}} \cong 0.1$. Also, in fig. 4 , the asymptotics for the densities is compared with the numerical values, for $\gamma_{\mathrm{A}}=0.01$, as a function of $\eta$. In this figure, one can clearly see the crossover from BNE to BNE of the model. 


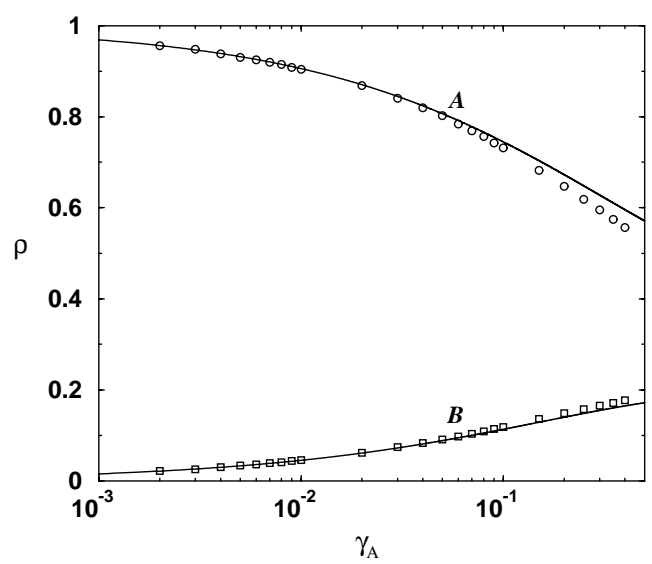

Fig. 3

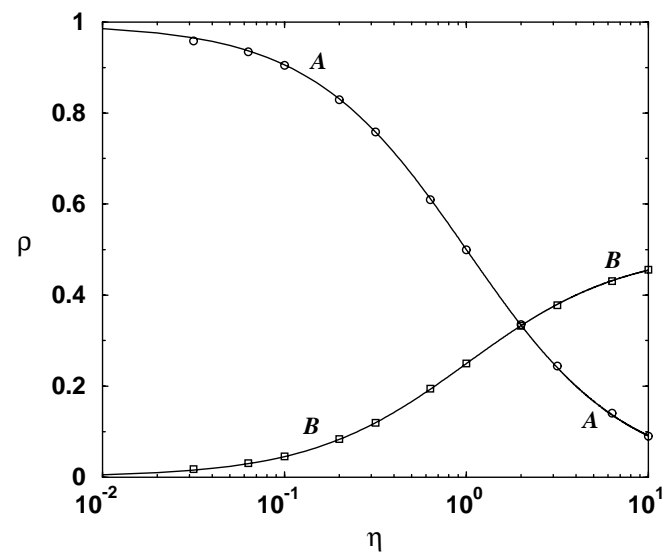

Fig. 4

Fig. 3 - Comparison between the numerical values of the steady densities, for particles A (circles) and particles B (squares), obtained by Monte Carlo simulation, and their corresponding analytical expression (solid line), eq. (10), for $\gamma_{\mathrm{A}}=\gamma_{\mathrm{B}}$. The agreement is quite good for $\gamma_{\mathrm{A}} \leq 0.1$.

Fig. 4 - Steady values of the densities of particles A (circles) and particles B (squares), for $\gamma_{\mathrm{A}}=0.01$, as a function of $\eta$. In the small $\eta$ region, $\rho_{\mathrm{A}} \gg \rho_{\mathrm{B}}$, while $\rho_{\mathrm{B}} \gg \rho_{\mathrm{A}}$ when $\eta$ is large.

In the region $\eta \ll 1$, we obtain $\rho_{\mathrm{A}} \rightarrow 1$ and $\rho_{\mathrm{B}} \rightarrow 0$, our system is full of small particles $\mathrm{A}$ (BNE). As the size effect is taken into account by means of the different facilitation rule, if both species are identical except for their size, it would be $\alpha_{\mathrm{a}}=\beta_{\mathrm{a}}$ and $\alpha_{\mathrm{d}}=\beta_{\mathrm{d}}$, i.e., $\gamma_{\mathrm{A}}=\gamma_{\mathrm{B}}$ and $\eta=\sqrt{\gamma_{\mathrm{A}}} \ll 1$. This is why no RBNE is observed with particles of the same material $[4,5]$.

On the other hand, when $\eta \gg 1$, it is $\rho_{\mathrm{A}} \rightarrow 0$ and $\rho_{\mathrm{B}} \rightarrow 1 / 2$. Now, the layer is full of big particles $\mathrm{B}$. The line separating these opposite behaviours can be determined by the condition $\rho_{\mathrm{A}}=\rho_{\mathrm{B}}$. To the lowest order, this occurs for $\eta=2$, i.e., $\gamma_{\mathrm{A}}=2 \sqrt{\gamma_{\mathrm{B}}}$. In order to understand why the parameter $\eta$ governs the transition from BNE to RBNE, let us consider the transitions consisting in the substitution of two particles A by one particle B. In table I, it is seen that the ratio of their effective rate $W_{\text {ef }}$ to those of their inverse transitions is $\eta^{2}$. In a low layer of a real granular system, the desorption rates of the grains should decrease with their mass density. In fact, a comparison with refs. $[15,16]$ indicates that $\eta$ plays in the model a role analogous to the ratio of the mass density of the big grains to the small ones in real granular systems and hydrodynamic models. Therefore, the region $\eta \geq 2$, for which the RBNE is expected, would correspond to large values of the mass density of the big grains, as compared with the mass density of the small ones. Then, the picture emerging from the model agrees with both numerical and experimental results $[15,16]$. Interestingly, in the model, the RBNE is accompanied by a non-trivial behaviour of the time evolution of the total density of particles $\rho_{\mathrm{A}}+\rho_{\mathrm{B}}$, which presents a maximum at a certain time $t_{\mathrm{c}}$. Roughly speaking, the total density increases until the time $t_{\mathrm{c}}$ for which $\rho_{\mathrm{A}}=\rho_{\mathrm{B}}$; for greater times $\rho_{\mathrm{B}}<\rho_{\mathrm{A}}$ and the total density decreases [19]. We are not aware of experimental data allowing to check this prediction.

In summary, a simple one-dimensional lattice model for a horizontal section of a granular binary mixture, near the bottom of its container, has been presented. The number of particles of this section of the system is not conserved, when it is externally perturbed. This is a main difference with other models for segregation, in which the number of particles is constant [21, 22]. In our model, small (big) particles need one (both) of its nearest-neighbour sites to be empty in order to adsorb on or desorb from the lattice. The system is submitted to 
a tapping process, so that in each free relaxation the system evolves until it gets stuck in a metastable state. For weak vibration, an effective dynamics connecting the metastable states has been derived. The steady-state distribution is consistent with Edwards' theory of powders, and approximate analytical expressions for the steady densities have been obtained. The steady distribution is characterized by the fugacities of both species, which are related to their adsorption and desorption dynamical rates. There are two well-defined limit behaviours. Depending on the relative values of the fugacities, the layer exhibits dominance of small particles (BNE) or big particles (RBNE).

We acknowledge support from the Ministerio de Ciencia y Tecnología (Spain) through Grant No. BFM2002-00307 (partially financed by FEDER funds).

\section{REFERENCES}

[1] Jaeger H. M., Nagel S. R. and Behringer R. P., Rev. Mod. Phys., 68 (1996) 1259.

[2] Kadanoff L. P., Rev. Mod. Phys., 71 (1999) 435.

[3] Knight J. B., Frandich C. G., Lau C. N., Jaeger H. M. and Nagel S. R., Phys. Rev. E, 51 (1995) 3957.

[4] Williams J. C., Powder Technol., 15 (1976) 245.

[5] Rosato A., Strandburg K. J., Prinz F. and Swendsen R. H., Phys. Rev. Lett., 58 (1987) 1038.

[6] Brey J. J., Prados A. and Sánchez-Rey B., Phys. Rev. E, 60 (1999) 5685; Physica A, 275 (2000) 310.

[7] Barrat A., Kurchan J., Loreto V. and Sellitto M., Phys. Rev. Lett., 85 (2000) 5034; Phys. Rev. E, 63 (2001) 051301.

[8] Lefevre A. and Dean D. S., J. Phys. A, 34 (2001) L213; Dean D. S. and Lefevre A., Phys. Rev. Lett., 86 (2001) 5639.

[9] Berg J. and Mehta A., Adv. Complex Syst., 4 (2001) 309.

[10] Prados A. and Brey J. J., Phys. Rev. E, 66 (2002) 041308.

[11] Berg J., Franz S. and Sellitto M., Eur. Phys. J. B, 26 (2002) 349.

[12] Ben-Naim E., Knight J. B. and Nowak E. R., J. Chem. Phys., 100 (1996) 6778; Kolan A. J., Nowak E. R. and Tkachenko A. V., Phys. Rev. E, 59 (1999) 3094.

[13] Edwards S. F. and Oakeshott R. B. S., Physica A, 157 (1989) 1080; Mehta A. and EdWARds S. F., Physica A, 157 (1989) 1091.

[14] Möbius M. E., Lauderdale B. E., Nagel S. R. and Jaeger H. M., Nature, 414 (2001) 270.

[15] Hong D. C., Quinn P. V. and Luding S., Phys. Rev. Lett., 86 (2001) 3423; Bотн J. A. and Hong D. C., Phys. Rev. Lett., 88 (2002) 124301.

[16] Breu A. P. J., Ensner H.-M., Kruelle C. A. and Rehberg I., Phys. Rev. Lett., 90 (2003) 014302.

[17] Fredrickson G. H. and Andersen H. C., Phys. Rev. Lett., 53 (1984) 1244.

[18] Prados A., Brey J. J. and Sánchez-Rey B., Physica A, 284 (2000) 277.

[19] Prados A. and Brey J. J., unpublished.

[20] van Kampen N. G., Stochastic Processes in Physics and Chemistry (North-Holland, Amsterdam) 1992.

[21] Nicodemi N., Fierro A. and Coniglio A., Europhys. Lett., 60 (2002) 684.

[22] Braun J., Phys. Rev. E, 64 (2001) 011307. 